\section{Iron deficiency and iron deficiency anaemia in elderly patients with chronic heart failure}

\author{
Lorenzo Palleschi, ${ }^{1}$ Eleonora Nunziata ${ }^{2}$ \\ ${ }^{1}$ Unit of Geriatrics, A.O. San Giovanni- \\ Addolorata, Roma; ${ }^{2}$ Unit of Medicine, \\ Asl RM4, Roma, Italy
}

\begin{abstract}
Iron deficiency affects up to $50 \%$ of heart failure patients, and is associated with poor quality of life, impaired exercise tolerance, and mortality independent of haematopoietic effects in this patient population. Iron absorption from oral iron preparations is generally poor, with slow and often inefficient iron repletion; moreover, up to $60 \%$ of patients experience gastrointestinal side effects. These problems may be exacerbated in heart failure due to decreased gastrointestinal absorption and poor compliance due to pill burden. Intravenous (i.v.) iron sucrose has consistently been shown to improve exercise capacity, cardiac function, symptom severity, and quality of life. Similar findings were observed recently for i.v. ferric carboxymaltose in patients with systolic heart failure and impaired left ventricular ejection fraction. I.v. iron therapy may be better tolerated than oral iron. Routine diagnosis and management of iron deficiency in patients with symptomatic heart failure regardless of anaemia status is advisable, and, based on current evidence, prompt intervention using i.v. iron therapy should now be considered.
\end{abstract}

\section{Introduction}

Disorders of iron metabolism are common human pathologic conditions. Iron deficiency (ID), defined as low body iron with or without anaemia, is estimated to affect 2 billion people. Iron deficiency anaemia is the most prevalent anaemia worldwide roughly affecting 1 of 8 persons, with the highest prevalence in developing countries. ${ }^{1}$

Iron deficiency anemia (IDA) affects a large part of adult and elderly patients admitted to Geriatric and Internal Medicine Units. In high-income countries, iron deficiency anaemia, usually with the exception of groups with increased iron needs (young children and adolescents, pregnant and postpartum women), may lead to pathologic conditions associated with poor iron absorption and/or chronic blood loss.

IDA is a microcytic anaemia, which defines a decrease mean red cell volume as a consequence of reduced hemoglobin $(\mathrm{Hb})$ production. ID with or without anaemia accounts for approximately $80 \%$ of microcytosis; whereas, rare inherited defects of iron metabolism, of globin chains and hemesynthesis account globally for almost $20 \%$ of microcytic anaemias.

By excluding inherited red cells disorders, ID seems to be the main cause of increased years life lived with disability observed in all ages and in both gender by global burden disease 2010 studies. This results in low patient quality of life (QoL) and increase risk of developing severe organ complications with growing cost for National health systems. ${ }^{2}$

International guidelines agree on performing key blood tests for diagnosing the IDA. Up to now serum ferritin and transferrin saturation are the key tests in early decision making process to identify IDA. Marker of inflammation such as C-reactive protein has to be evaluated in order to exclude a possible co-existing chronic inflammation disease. Based on these parameters, we identified i) an absolute iron deficiency, when the total body iron stores are depleted; and ii) a functional iron deficiency, when the body iron mobilization is altered and does not meet the iron demand for the erythropoiesis. ${ }^{3}$

\section{Anaemia in elderly}

There is some debate on which $\mathrm{Hb}$ threshold should be used to define anaemia in the general population and particularly in elderly individuals. In many studies, anaemia has been defined according to the World Health Organization (WHO) criteria $^{4}$ as a $\mathrm{Hb}$ level $<13 \mathrm{~g} / \mathrm{dL}$ in men and $<12 \mathrm{~g} / \mathrm{dL}$ in women, respectively. However, these criteria have been criticized since they were based on statistical distributions (i.e., equivalent to two standard deviations below the mean) in reference samples that did not include individuals $>65$ years of age, making unfeasible their application to older individuals. ${ }^{5}$ Since Hb values in apparently healthy elderly individuals are generally lower than those in younger adults and the differences between males and females tend to disappear with aging, a $\mathrm{Hb}$ value $<12$ $\mathrm{g} / \mathrm{dL}$ is now commonly considered indicative of anaemia in elderly of both sexes. ${ }^{6}$

Anaemia is a common, multi-factorial condition in elderly and deeply influences patient mortality and quality of life by i)
Correspondence: Eleonora Nunziata, Unit of Medicine, Asl RM4, Roma, Italy.

E-mail: ele.nunziata@gmail.com

Key words: Iron deficiency; chronic heart failure; elderly.

Conference presentation: paper presented at $31^{\circ}$ Congresso Nazionale SIGOT - Genova, June 8-9, 2017.

Received for publication: 27 October 2017. Accepted for publication: 9 November 2017.

This work is licensed under a Creative Commons Attribution-NonCommercial 4.0 International License (CC BY-NC 4.0).

(C) Copyright L. Palleschi and E. Nunziata, 2017 Licensee PAGEPress, Italy

Geriatric Care 2017; 3:7163

doi:10.4081/gc.2017.7163

decreasing physical performance and cognitive function; and ii) increasing number of falls and hospitalization. ${ }^{7}$

Approximately, one-third of the cases of anaemia in elderly can be ascribed to a chronic disease (inflammation and chronic kidney diseases), while one-third is due to nutrient deficiencies (folate, B12, and iron). ID, alone or in combination with deficiency of other nutrients, accounts for more than one-half of this group. The last third remains unexplained ${ }^{8}$.

In a cohort study of 1160 Italian subjects of 90 years and over $(90+$, mean age 93 years; age range 90-106 years) followed for 6 years survival, the following factors emerged as significant predictors of survival after 90 years of age: absence of cognitive impairment and physical disability, high hand grip strength scores and body mass index values, excellent/good self-reported health, high haemoglobin and total cholesterol levels and low creatinine levels. ${ }^{9}$

\section{Iron deficiency in heart failure}

Iron deficiency is common in patients with chronic heart failure, relates to disease severity, and is a strong and independent predictor of outcome. A study revealed that ID appears to have greater predictive power than anaemia, affecting half of the patients $(50 \%)$. Anaemic patients were more often iron deficient than non-anaemic patients (61.2\% vs $45.6 \%, \mathrm{P}<0.001)$. ID identifies those with an enhanced risk for death, independently of other well established predictors of outcome, including anemia. ${ }^{10}$ Anaemia and ID are common in $\mathrm{CHF}$ 
patients and both are reported to have negative consequences..$^{10,11}$ In the past, the presence of ID was only considered clinically relevant in the presence of anaemia. Although ID can often be detected in anaemic CHF patients, almost one third of the patients with $\mathrm{CHF}$ and without anaemia can present ID. ${ }^{10,11}$ Iron is an important part in many human biochemical reactions. Besides its role in hemoglobin synthesis, iron is essential to oxygen transport and storage, oxidative metabolism in skeletal and heart muscle, immune system regulation and protein transcription. Because of its central role in oxygen transport and delivery, in the patients with systolic CHF, the presence of ID as well as reduced serum ferritin is associated with impaired exercise tolerance, independently of the other parameters traditionally linked with poor exercise capacity (including hemoglobin level). ${ }^{11}$

A study sought to determine the characteristics and long-term prognosis of anaemia in ambulatory patients with chronic heart failure, reavealed that anaemia is underrecognized and underevaluated, and the presence of persistent anaemia conferred poorer survival in patients with heart failure when compared with that of incident, resolved, or no anaemia. ${ }^{12}$

\section{How to treat iron deficiency}

The treatment of iron deficiency is based on supplementation, as oral iron or intravenous iron administration.

Oral iron therapy is relatively inexpensive and widely used, but the absorption of iron is low. Oral iron is most frequently given in the form of ferrous (Fe[II]) salts, such as ferrous sulfate. Oxidation of $\mathrm{Fe}$ (II) to $\mathrm{Fe}(\mathrm{III})$ can provoke oxidative damage at the mucosal boundary, resulting in local toxicity which leads to gastrointestinal side effects in up to $60 \%$ of patients. These include complications such as constipation, dyspepsia, nausea, diarrhoea, and heartburn, which are particularly frequent with ferrous sulfate preparations and often lead to poor compliance. The effects of oxidative stress can be avoided by use of the oral preparation iron[III]-polymaltose complex, which includes a polymaltose shell for stability so that iron is released in a controlled manner and non-transferrin-bound iron does not increase. The structure of the iron[III]-polymaltose complex also minimizes the interactions with food or concomitant medications that can affect uptake of iron from ferrous salts. The choice on iron supplementation is based on Hb levels, the tolerance to oral iron supplementation and the presence of concomitant disease, which might affect iron absorption. The failure of oral iron supplementation might be related to either low patient adherence to the therapy (i.e.: treatment discontinuation, low tolerance mainly due to GI symptoms) or to true refractoriness.

Oral iron supplementation should be maintained for 3 to 6 months to replete the iron stores and normalize ferritin level.

Intravenous iron administration is definitively more effective in correction of ID since it by-passes the iron absorption step. Although its costs are higher than oral iron supplementation, it offers several advantages such as i) rapid repletion of iron stores; ii) single dose sufficient for most of the new i.v. formulation with a reduction in hospital visits. This latter is limited to the more recently developed molecules, which are characterized by a core that contains the iron salt surrounded by a shell, allowing the reticuloendothelial system to process and release iron for erythropoiesis and storage. Although adverse events have been reported for i.v. formulation, the cost-benefit ratio is in favor of i.v. supplementation.

A series of i.v. iron complexes has been developed which enclose an iron-containing core within a carbohydrate shell, stabilizing the structure and controlling the release of iron. The first complex, iron sucrose, was followed by low and high molecular weight iron dextran products and ferric gluconate. More recently, ferric carboxymaltose, iron isomaltoside 1000 , and ferumoxytol have become available. These are more thermodynamically stable, with a low reduction potential, and permit administration of a much higher dose of iron in a single administration.

In clinical trials, i.v. iron supplementation and correction of anaemia improve: i) New York Heart Association (NYHA) functional classes; ii) exercise capacity and iii) patients QoL. Thus, iron supplementation should be always considered as part of clinical management of CHF patients. ${ }^{13}$

Similar findings were observed recently for i.v. ferric carboxymaltose in patients with systolic heart failure and impaired left ventricular ejection fraction (LVEF) in the double-blind, placebo-controlled FAIR$\mathrm{HF}^{14}$ and CONFIRM-HF trials. ${ }^{15}$

The European Society of Cardiology Guidelines for heart failure 2012 recommend a diagnostic work-up for iron deficiency in patients with suspected heart failure. ${ }^{16}$ In the newer 2016 guidelines of the ESC for the diagnosis and treatment of HF, iron deficiency may contribute to muscle dysfunction in $\mathrm{HF}$ and causes anaemia. ${ }^{17}$

Certain characteristics of patients with heart failure, which can limit intestinal absorption of oral iron preparations can be particularly pronounced and therefore i.v. iron is the preferred option. First, patients with heart failure are usually receiving multiple medications for heart failure and frequently for one or more comorbid conditions. Secondly, various factors can contribute to abnormal intestinal morphology and function in heart failure and therefore inhibit iron absorption, such as oedema of the absorptive mucosa. Other changes can include overactivation of the sympathetic nervous system, the effect of concomitant medications such as $\mathrm{H} 2$ blockers, and complications of co-morbid conditions such as diabetes-related gastroparesis.

The median required dose of iron to achieve iron repletion in iron-deficient patients with heart failure is $1000 \mathrm{mg}$. In patients with iron deficiency anaemia, the rate of iron bioavailability from a therapeutic dose of oral $100 \mathrm{mg}$ ferrous sulfate, the most commonly used oral iron preparation, and iron[III]-polymaltose complex, is $\sim 10 \%$. Given that a typical dose of ferrous sulfate is $100-200 \mathrm{mg}$ iron/day, in the best case scenario, a patient who tolerates treatment of $200 \mathrm{mg} /$ day might absorb only 20 $\mathrm{mg}$ of iron and thus require 50 days to correct the iron deficit. In a worst case scenario, given that patients with chronic heart failure may have as little as half the usual rate of active carrier-mediated intestinal absorption capacity, a patient who can tolerate up to $100 \mathrm{mg}$ iron/day might absorb only $5 \%(5 \mathrm{mg})$. It would thus require 200 days to replenish iron stores. In practice, correction of iron deficiency for heart failure patients will often take longer due to nonadherence or interruption due to gastrointestinal side effects, or missed doses due to the high pill burden. Therefore, replenishment of iron stores with oral iron can be expected to require $>6$ months. In contrast, one or two doses of i.v. iron therapy can achieve rapid repletion. I.v. administration, however, places additional demands on the treatment centre.

In the IRONOUT study high-dose of oral iron (Fe) polysaccharide did not improve exercise capacity, as measured by change in peak VO2 (oxygen uptake) by CPET (Cardiopulmonary Exercise Testing), of a broad population of patients with $\mathrm{HFrEF}$ (Heart Failure with Reduced Ejection Fraction) and Fe deficiency at 16 weeks. ${ }^{18}$

\section{Conclusions}

Iron deficiency is widespread in patients with heart failure. Iron is poorly absorbed from oral preparations, compounded by chronic inflammation and other 
contributing factors in patients with heart failure, which delays or prohibits iron replenishment. Evidence for a clinical benefit using oral iron preparations in heart failure is lacking. Clinical studies have instead focused on the use of i.v. iron, which bypasses the problem of gastrointestinal absorption and poor compliance.

Early studies using iron sucrose consistently showed that correction of iron deficiency improved exercise capacity, cardiac function, symptom severity, and quality of life. ${ }^{13}$ These studies were recently confirmed in the large 6-month FAIR-HF study, ${ }^{14}$ where functional capacity, symptoms, and quality of life rapidly increased in patients with heart failure of NYHA class II-III and impaired LVEF in comparison with placebo treatment. Longer term data were provided by the CONFIRM-HF trial, ${ }^{15}$ in which symptomatic, iron-deficient patients with heart failure showed a sustained improvement in functional capacity, symptoms, and quality of life, and reduced risk of hospitalization due to worsening heart failure over 1 year of i.v. iron therapy.

The ESC Guidelines point out that iron deficiency may contribute to muscle dysfunction in $\mathrm{HF}$ and causes anaemia and ferric carboxymaltose may be considered for treatment of these patients. ${ }^{16,17}$ The structures of new i.v. preparations permit far larger doses of iron to be administered safely in a single visit. Routine diagnosis and management of iron deficiency in patients with symptomatic heart failure, regardless of anaemia status, and prompt intervention using i.v. iron therapy should now be considered.

\section{References}

1. Kassebaum NJ, Jasrasaria R, Naghavi $\mathrm{M}$, et al. A systematic analysis of global anemia burden from 1990 to 2010 . Blood 2014;123:615-24.

2. Murray CJ, Vos T, Lozano R, et al. Disability-adjusted life years (DALYs) for 291 diseases and injuries in 21 regions, 1990-2010: a systematic analysis for the global burden of disease study 2010. Lancet 2012;380:2197-223.

3. Thomas DW, Hinchliffe RF, Briggs C, et al. Guideline for the laboratory diagnosis of functional iron deficiency. $\mathrm{Br} \mathrm{J}$ Haematol 2013;161:639-48.

4. Blanc B, Finch CA, Hallberg L, et al. Nutritional anaemias report of a WHO scientific group. World Health Organ Tech Rep Ser 1968;405:1-40.

5. Mindell J, Moody A, Ali A, et al. Using longitudinal data from the Health Survey for England to resolve discrepancies in thresholds for haemoglobin in older adults. Br J Haema-tol 2013;160: 368-76.

6. Izaks GJ, Westendorp RG, Knook DL, et al. The definition of anemia in older person. JAMA 1999;281:1714-7.

7. Culleton BF, Manns BJ, Zhang J, et al. Impact of anemia on hospitalization and mor-tality in older adults. Blood 2006; 107:3841-6.

8. Guralnik JM, Eisenstaedt RS, Ferrucci, et al. Prevalence of anemia in persons 65 years and older in the United States: evidence for a high rate of unexplained anemia. Blood 2004;2263-8.

9. Cevenini E, Cotichini R, Stazi MA, et al. Health status and 6 years survival of 552 90+ Italian sib-ships recruited within the EU Project GEHA (GEnetics of Healthy Ageing). Age (Dordr) 2014;36: 949-66.

10. Ezekowitz JA, McAlister FA, Armstrong PW. Anemia is common in heart failure and is associated with poor outcomes: insights from a cohort of 12,065 patients with new-onset heart failure. Circulation 2003;107:223-5.

11. Jankowska EA, Rozentryt P, Witkowska A, Nowak J. Iron deficiency: an ominous sign in patients with systolic chronic heart failure. Eur Heart J 2010; 31:1872-80.

12. Wilson Tang WH, Tong W, Jain A, et al. Anemia in ambulatory heart failure patients mortality risk ratios by anemia status at baseline and follow-up. JACC 2008;51:5.
13. Anker SD, Comin Colet J, Filippatos G. Ferric carboxymaltose in patients with heart failure and iron deficiency. N Engl J Med 2009;361:2436-48.

14. Anker SD, Colet JC, Filippatos G, et al. FAIR-HF committees and investigators. Ra-tionale and design of Ferinject assessment in patients with IRon deficiency and chronic Heart Failure (FAIR-HF) study: a randomized, placebo-controlled study of intravenous iron sup-plementation in patients with and without anaemia. Eur J Heart Fail 2009; $11: 11$.

15. Ponikowski $P$, van Veldhuisen DJ, Comin-Colet $J$, et al. Rationale and design of the CONFIRM-HF study: a double-blind, randomized, placebocontrolled study to assess the ef-fects of intravenous ferric carboxymaltose on functional capacity in patients with chronic heart failure and iron deficiency. ESC Heart Fail 2014;1:52-8.

16. McMurray J, Adamopoulos S, Anker $\mathrm{SD}$, et al. ESC guidelines for the diagnosis and treatment of acute and chronic heart failure 2012: the task force for the diagnosis and treat-ment of acute and chronic heart failure 2012 of the European Society of Cardiology. Developed in collaboration with the Heart Failure Association (HFA) of the ESC. Eur J Heart Fail 2012;14:803-69.

17. Ponikowski P, Voors AA, Anker SD, et al. 2016 ESC Guidelines for the diagnosis and treatment of acute and chronic heart failure: The Task Force for the diagnosis and treat-ment of acute and chronic heart failure of the European Society of Cardiology (ESC). Developed with the special contribution of the Heart Failure Association (HFA) of the ESC. Eur Heart J 2016;37:2129-200.

18. Lewis GD, Malhotra I, Hernandez AF, et al. Effect of oral iron repletion on exercise capacity in patients with heart failure with reduced ejection fraction and iron deficiency: the IRONOUT HF randomized clinical trial. JAMA 2017; 317:1958-66 